\title{
Efficient MAC in Cognitive Radio Systems: A Game-Theoretic Approach
}

\author{
Márk Félegyházi, Member, IEEE, Mario Čagalj, Member, IEEE, and Jean-Pierre Hubaux, Fellow, IEEE
}

\begin{abstract}
In this paper, we study the problem of efficient medium access control (MAC) among cognitive radio devices that are equipped with multiple radios and thus are capable of transmitting simultaneously at different frequencies (channels). We assume that radios contend on each channel using the $\mathrm{Car}$ rier Sense Multiple Access with Collision Avoidance (CSMA/CA) protocol. We study two MAC problems: (i) the allocation of the available channels among radios, and (ii) the optimal usage of each allocated channel by the radios occupying it. Both problems are studied in a game-theoretic setting, where devices aim to selfishly maximize their share of the available bandwidth. As for the first problem, we show that the "price of anarchy" is close to 1 , that is, Nash equilibria imply nearly system optimal allocations of the available channels. For the second problem, we design a game such that it admits a unique Nash equilibrium that is is both fair and Pareto-optimal. Furthermore, we propose simple mechanisms that enable selfish cognitive radio devices not only to coordinate efficiently on the available channels but also to optimally use every single allocated channel.
\end{abstract}

Index Terms-Cognitive radio networks, MAC, CSMA/CA, cooperation, game theory, Nash equilibrium, Pareto-optimality.

\section{INTRODUCTION}

W IRELESS communications rely on the frequency spectrum as a fundamental resource. With the increasing demand from upcoming wireless communication technologies, the available frequency spectrum became scarce. This initiated the government agencies to carefully control the access to this precious resource. First, this control was exercised based on a first-come-first-served basis and then based on spectrum auctions. Yet, this practice of fixed frequency assignment is not flexible enough to maintain the pace with the advancement of wireless communication technologies. Many frequency bands, that are previously assigned, are almost unused, whereas some networks serve an enormous communication demand.

Cognitive radio technology [1], [2] promises to mitigate the inflexibility of existing spectrum regulations. Cognitive radio devices are capable of adapting to their environment

Manuscript received March 9, 2008; revised September 2, 2008; accepted October 25, 2008. The associate editor coordinating the review of this paper and approving it for publication was G. Xue.

M. Félegyházi is with the International Computer Science Institute (ICSI) in Berkeley, USA (e-mail: mark@ icsi.berkeley.edu).

M. Čagalj is with the Faculty of Electrical Engineering, Mechanical Engineering and Naval Architecture (FESB), University of Split, Croatia (email: mario.cagalj@fesb.hr).

J.-P. Hubaux is with EPFL, Switzerland (e-mail: jeanpierre.hubaux@epfl.ch).

The work presented in this paper was supported (in part) by the National Competence Center in Research on Mobile Information and Communication Systems (NCCR-MICS), a center supported by the Swiss National Science Foundation under grant number 5005-67322.

Digital Object Identifier 10.1109/TWC.2009.080284 by selecting the unused frequencies and transmission powers for communication. This enables users (or operators) with cognitive radios to communicate using frequencies previously assigned to other technologies called primary users without interfering with them.

The emergence of cognitive radios can result in a flexible spectrum management. However, cognitive radios are strategic by definition, meaning that they implement an optimization strategy that adapts to the best communication possibilities. This selfish optimization of cognitive radio devices might have a negative effect on the overall network performance. Therefore, cognitive radio protocols have to be assessed in a non-cooperative setting.

In this paper, we study the problem of non-cooperative medium access of cognitive radio devices equipped with multiple radio adapters. Since primary users are non-strategic (typically they use older technologies that cannot even detect the operation of cognitive radios), we focus exclusively on the selfish game between cognitive radio devices. We assume that the available frequency band is split into orthogonal channels. We assume that channel access is based on the CSMA/CA protocol. This protocol is the most popular in currently upcoming technologies and we anticipate that first cognitive radio systems will rely on this technology as well.

We define a two-tier medium access control (MAC) game in which selfish cognitive radio devices first coordinate to achieve a channel allocation of their radios over the available channels assuming that the bandwidth of each channel will be fairly allocated later. Then, they optimize the channel access parameters of the CSMA/CA protocol of their radios on each channel to indeed achieve this fair allocation. Selfish users have two choices to obtain more throughput on any channel $c$ : (i) they either use several radios on $c$ to exploit the per radio fairness property of the CSMA/CA backoff algorithm or (ii) they can subvert this backoff algorithm by cheating with the backoff parameter. We explore both options in the MAC game and accordingly we split it into two sub-games: the channel allocation (CA) game and the multiple access (CSMA/CA) game.

Figure 1 shows the unfolding of the MAC game. Let $T_{C A}^{c o n v}$ and $T_{C A}$ denote the convergence time and the total subgame time of the channel allocation game. Similarly, $T_{C S M A / C A}^{c O n v}$ and $T_{C S M A / C A}$ denote the convergence time and total time of the multiple access subgame. We assume that the devices start optimizing their channel access parameters upon the convergence of the channel allocation protocol. Thus the channel allocation game ends with the convergence to a stable 


\begin{tabular}{|c|c|c|c|}
\hline CA game & $\begin{array}{c}\text { per channel } \\
\text { CSMA/CA game }\end{array}$ & CA game & $\begin{array}{c}\text { per channel } \\
\text { CSMA/CA game }\end{array}$ \\
\hline$T_{C A}(1)$ & $T_{\text {CSMA }}(1)$ & $T_{C A}(2)$ & $T_{\text {CSMA }}(2)$ \\
\hline
\end{tabular}

Fig. 1. The game played by the players: the players alternate between the channel allocation (CA) game and the per channel multiple access (CSMA/CA) game.

state (i.e., $T_{C A}^{c o n v}=T_{C A}$ ). Then, the players optimize the parameters of their CSMA/CA protocol within $T_{C S M A / C A}^{c o n v}$. Following the convergence in the CSMA/CA game, the devices communicate efficiently until a new CA game starts (i.e., $\left.T_{C S M A / C A}^{c O n v}<T_{C S M A / C A}\right)$. We show in Section VII that both protocols converge quickly to a stable operation. Note that the two-tier procedure is repeated from time to time to cope with the potential dynamic nature of the network.

The main contribution of this paper is to extend the protocols presented in [3] and [4]; and to establish the interaction between the two games described therein. As we show in Section VII-C, the CA game discourages players to apply excessive cheating in the CSMA/CA game. Analogously, the CSMA/CA game not only optimizes the channels' usage, but also provides an incentive to the players in the CA game to behave "optimally".

The paper is organized as follows. In Section II, we survey related work. In Sections III and IV, we present the system and game-theoretic models, respectively. In Section V, we present the channel allocation game, and Section VI studies the CSMA/CA game. In Section VII, we present two simple mechanisms to achieve the efficient Nash equilibria. We conclude in Section VIII.

\section{RELATED WORK}

Cognitive radio is a novel technology that enables flexible spectrum utilization. Mitola [2] discusses the potential of the cognitive radio architecture and outlines potential radioetiquette protocols. Haykin [1] gives an overview of the stateof-the-art in cognitive radio. Mähönen [5] discusses trends in the emergence of the cognitive radio technology. More recently, Wang et al. [6] propose an iterative water-filling algorithm for power-control and channel assignment in cognitive radio networks. Zou et al. [7] study a QoS-aware dynamic spectrum allocation protocol and propose two game-based algorithms.

Recently, several researchers have considered devices using multiple radios, notably in mesh networks (for a survey on mesh networks, see [8]). In the multi-radio communication context, channel allocation and access also became crucial topics. Related work on multi-radio medium access includes, but is not restricted to [9]-[11]. Mishra et al. [12] propose a channel allocation method for wireless local area networks (WLANs) based on weighted graph coloring. Zheng and Cao [13] present a rule-based spectrum management scheme for cognitive radios.

In all the related work cited so far, the authors assumed that the radio devices cooperate to achieve a high system performance. But this assumption might not hold, as the users of these devices are usually selfish and they want to maximize their own performance without necessarily respecting the system objectives. Game theory provides a straightforward tool to study multiple access problems in competitive wireless networks and has been applied to the CSMA/CA protocol [3], [14] and to the Aloha protocol [15]. Furthermore, a fixed channel allocation game was presented in [16] based on graph coloring. But, the model in [16] does not apply to multiradio devices. For cognitive radio networks, the authors of [17] propose a dynamic channel allocation scheme based on a potential game. In addition, they suggest another technique based on machine learning with different utility functions. Neel et al. [18] suggest to study cognitive radio behavior using S-modular and potential games. Cao and Zheng [19] propose distributed spectrum allocation in cognitive radio networks based on local bargaining.

\section{System Model}

We assume that the available frequency band is divided into orthogonal channels of the same bandwidth using the FDMA method. We denote the set of available orthogonal channels by $\mathcal{C}$. We assume that there is a mechanism that enables the wireless devices to use multiple channels to communicate at the same time (as it is shown in [9] for example). Moreover, the devices implement the Carrier Sense Multiple Access with Collision Avoidance (CSMA/CA) protocol to resolve contention at the Medium Access Control (MAC) layer on each channel [20]. CSMA/CA protocols rely on the random deferment of packet transmissions for the efficient use of a shared wireless channel among many devices in a network; this class of MAC protocols is one of the most popular for wireless networks. We further assume that each radio of each device has a unique MAC layer identifier (the MAC address) which is achieved by an appropriate MAC layer authentication.

In our model, pairs of users want to communicate with each other over a single hop. We assume that each user participates in only one such communication session and we denote the set of communicating pairs by $\mathcal{N}$. We assume that $\mathcal{N}$ is finite. Each user owns a device equipped with $k$ radio adapters, all having the same communication capabilities. We assume that:

$$
k \leq|\mathcal{C}|
$$

This assumption is reasonable, because devices using the 802.11 MAC protocol are typically equipped with fewer radios than the total number of channels.

The communication links between two devices are bidirectional and devices always have some packets to exchange. Due to the bidirectional communication links, the sender and the receiver are able to coordinate and thus to select the same channels to communicate. We assume that each device can hear the transmissions of every other device if they are 


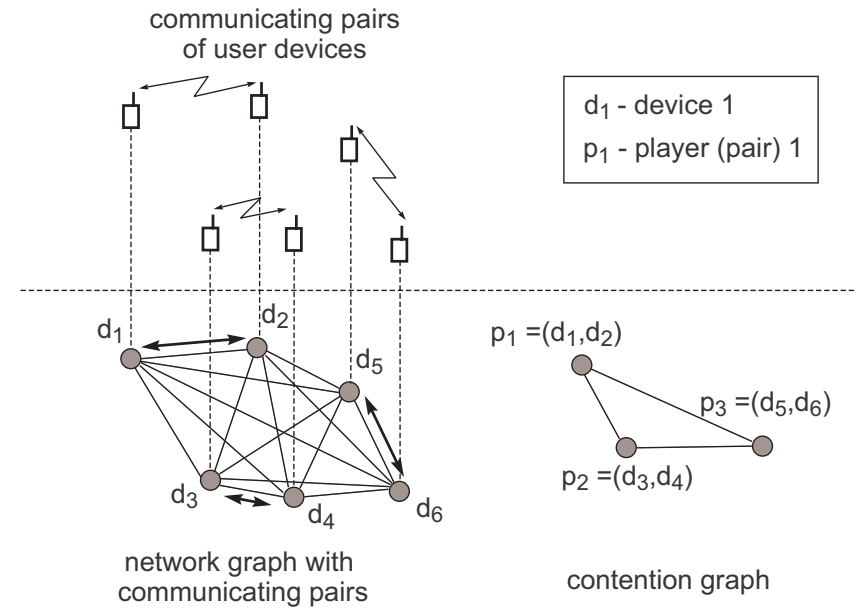

Fig. 2. A network graph for 3 pairs of users (i.e., $|\mathcal{N}|=3$ ) with the corresponding contention graph.

using the same channel. This means that they reside in a single collision domain. We make this assumption to avoid the hidden terminal problem described for example in [20]. This assumption implies that the channels have roughly the same radio characteristics.

From the network topology and the set of communication pairs, we can construct a contention graph, as described in [21] for example. Note that the notion of contention graph was introduced for single channel systems and we adapt it for the multi-channel scenario. We show an example in Figure 2.

\section{GAme-Theoretic Model}

We model the medium access problem in a two-tier medium access control (MAC) game as follows. We refer to any communicating pair of devices as a selfish player $i$, whose objective is to maximize his total throughput $r_{i}$ in the network. We will use the term "selfish player" to denote both the communicating pair of devices and the communication link between them. The players first coordinate to achieve a channel allocation of their radios over the available channels in $\mathcal{C}$ assuming a fair access on a single channel and then they optimize the channel access parameters of the CSMA/CA protocol of their radios on each channel. Accordingly, we split the MAC game into two sub-games: the channel allocation (CA) game and the multiple access (CSMA/CA) game.

The strategy $s_{i}$ of player $i$ defines his decisions in the game taking the decisions of other players into account. In the CA game, the strategy of player $i$ defines the number of radios player $i$ uses in each channel. In the CSMA/CA game, the strategy defines how assertively player $i$ contends for the available bandwidth on a specific channel by adjusting the backoff window parameter in the CSMA/CA protocol implemented in his radios. We provide more details in Sections V and VI. We denote the strategy space of player $i$ by $\mathcal{S}_{i}$. The strategies of the players define the strategy profile $s=\left\{s_{1}, \ldots, s_{|\mathcal{N}|}\right\}$. We denote all players other than player $i$ by " $-i$ " and their strategy profile by $s_{-i}=\left\{s_{1}, \ldots, s_{i-1}, s_{i+1} \ldots, s_{|\mathcal{N}|}\right\}$.

We assume that the players are rational and their objective is to maximize their payoffs in the network. We denote the payoff of player $i$ by $u_{i}$. We assume that each player $i$ wants to maximize his total throughput or bitrate $\left(r_{i}\right)$ in the system and thus his payoff function is written as follows:

$$
u_{i}=r_{i}=\sum_{c \in \mathcal{C}} r_{i, c}
$$

where $r_{i, c}$ is the throughput achieved by player $i$ on channel $c$.

We further assume that the total available throughput $r_{c}=\sum_{i} r_{i, c}$ on a channel $c$ (i.e., the sum of the achieved throughputs of all players on channel $c$ ) is a non-increasing function of the number of radios deployed on $c$. In theory, $r_{c}$ is independent of the number of radios on $c$ for the CSMA/CA protocol [22]. In practice, the backoff window values used in the CSMA/CA protocol implementation (e.g., in the 802.11 standard) are not optimal; and due to packet collisions, $r_{c}$ becomes a decreasing function of the number of radios on $c$.

To characterize stability in the MAC game, we introduce the concept of Nash equilibrium [23], [24].

Definition 1: The strategy profile $s^{*}=\left\{s_{1}^{*}, \ldots, s_{|\mathcal{N}|}^{*}\right\}$ defines a Nash Equilibrium (NE), if for each player $i$ the following condition holds:

$$
u_{i}\left(s_{i}^{*}, s_{-i}^{*}\right) \geq u_{i}\left(s_{i}^{\prime}, s_{-i}^{*}\right)
$$

for every strategy $s_{i}^{\prime} \in \mathcal{S}_{i}$.

This means that in a Nash equilibrium, none of the users can unilaterally change his strategy to increase his payoff. A Nash equilibrium is often inefficient from the system's point of view. We characterize the efficiency of the solution by the concept of Pareto-optimality.

Definition 2: The strategy profile $s^{p o}$ is Pareto-optimal if $\nexists s^{\prime}$ such that:

$$
u_{i}\left(s^{\prime}\right) \geq u_{i}\left(s^{p o}\right), \forall i
$$

with strict inequality for at least one player $i$.

This means that in a Pareto-optimal strategy profile $s^{p o}$ one cannot improve the payoff of any player $i$ without decreasing the payoff of at least one other player $j$.

We can characterize the efficiency of a wireless system using the concept of the price of anarchy [25].

Definition 3: The price of anarchy (POA) is the ratio between the sum of the payoffs achieved by the players using the system- (Pareto-) optimal strategy profile and in the worst-case Nash equilibrium.

In the analysis (Sections V and VI), we assume that the games are of perfect information, meaning that the players know the other players, their potential strategies and their payoff functions. In addition, players are able to observe the actions of other players. We will relax these assumptions in Section VII and design distributed convergence mechanisms that rely only on local information.

\section{Channel Allocation Game}

In this section, we first extend the basic concepts introduced in Section IV and present the channel allocation (CA) game. Then, we show the existence of Nash equilibria and study their efficiency using the concept of the price of anarchy. We also propose a distributed algorithm to achieve the identified Nash equilibria and discuss its properties. We omit the proofs of the results in this paper due to space constraints, but they can be found in [26]. 


\section{A. Preliminaries}

First, we extend the MAC game model by adding some concepts that are specific to the $\mathrm{CA}$ game. The players are the pairs of devices as in the MAC game. In the CA game, we define the strategy of player $i$ as his channel allocation vector (i.e., that defines the number of his radios on each of the channels). We denote the number of radios used by player $i$ on channel $c$ by $k_{i, c}$. Let us recall the important assumption that the available number of radio adapters is smaller than the total number of channels, i.e., $k \leq|\mathcal{C}|$. Then we can express the strategy of $i$ as $s_{i}=\left\{k_{i, 1}, \ldots, k_{i,|\mathcal{C}|}\right\}$.

The intuition is that players should not use more than one radio on any channel $c$. Note however, that the 802.11 backoff algorithm provides per radio fairness. Thus, any user who uses multiple radios obtains a larger proportion of the total channel throughput without tampering with the 802.11 protocol parameters. In addition, sophisticated selfish users can manipulate with the backoff mechanism of 802.11 as well. We study this latter problem in Section VI.

We denote the set of channels used by player $i$ by $\mathcal{C}_{i}$. The total number of radios employed by player $i$ can be written as $k_{i}=\sum_{c \in \mathcal{C}} k_{i, c}$. Similarly, we can obtain the number of radios using a particular channel $k_{c}=\sum_{i \in \mathcal{N}} k_{i, c}$. The strategy vectors of all players defines the strategy matrix $S$ (i.e., the strategy profile).

As mentioned before, the players are rational and their objective is to maximize their payoff $u_{i}$ (i.e., the achieved throughput $r_{i}$ ) in the network. Recall that the total available throughput $r_{c}$ on a channel $c$ is a non-increasing function of the number of radios $k_{c}$ deployed on this channel. In addition, we assume that the channels have the same characteristics. Thus, we characterize the achievable throughput by the function $r\left(k_{c}\right)$. In the CA game, the players assume that the total throughput on channel $c$ will be equally shared among the radio adapters using that channel. This fair throughput allocation is indeed achieved as shown in Section VI in the CSMA/CA game. We can then express the payoff $u_{i}$ of player $i$ as:

$$
u_{i}=r_{i}=\sum_{c \in \mathcal{C}} r_{i, c}=\sum_{c \in \mathcal{C}} \frac{k_{i, c}}{k_{c}} \cdot r\left(k_{c}\right)
$$

\section{B. Nash Equilibria}

In this section, we study the existence of Nash equilibria in the CA game. We assume that the total number of radios in the system is higher than the number of channels (i.e., $|\mathcal{N}| \cdot k>|\mathcal{C}|$ ), otherwise there is not conflict in how to allocate these radios. If there is no conflict, then each channel is occupied by one radio, which is obviously a Pareto-optimal Nash equilibrium.

First, assuming that the above condition holds, we show the following intuitive result: a selfish player should use all of his radios in order to maximize his total throughput.

Lemma 1: If $S^{*}$ is a NE of the CA game, then $k_{i}=k, \forall i \in$ $\mathcal{N}$.

Let us now consider two arbitrary channels $b$ and $c$. Without loss of generality, we assume that there are more radios using channel $b$, meaning that $k_{b} \geq k_{c}$, and denote their difference by $\delta_{b, c}=k_{b}-k_{c}$. Furthermore, let us divide the channels in a channel allocation $S$ into three sets. We define the set of channels $\mathcal{C}_{\max }$ with the maximum number of radios, i.e., where $b \in \mathcal{C}_{\max }$ has $k_{b}=\max _{l \in \mathcal{C}} k_{l}$. Similarly, let us define the set of the least occupied channels $\mathcal{C}_{\text {min }}$, where $c \in \mathcal{C}_{\text {min }}$ has $k_{c}=\min _{l \in \mathcal{C}} k_{l}$. We denote the remaining set of channels by $\mathcal{C}_{\text {rem }}$.

In the following lemma, we show that in a Nash equilibrium, the difference in the total number of radios between any two channels cannot exceed one, i.e., $\mathcal{C}_{\text {rem }}=\{\emptyset\}$.

Lemma 2: If $S^{*}$ is a NE in the multi-radio channel allocation game, then $\delta_{b, c} \leq 1$ for all $b, c \in \mathcal{C}$.

Lemma 2 shows that in a NE only the sets $\mathcal{C}_{\text {max }}$ and $\mathcal{C}_{\text {min }}$ exist. This establishes an interesting property about NE: In fact, all NE channel allocations achieve load-balancing over the channels in $\mathcal{C}$. Based on Lemma 2, we express a set of sufficient conditions for the NE.

Using Lemma 2, we express a set of necessary and sufficient conditions for the NE.

Theorem 3: Assume that $|\mathcal{N}| \cdot k>|\mathcal{C}|$. Then a channel allocation $S^{*}$ is a NE if the two following conditions hold:

$\triangleright \delta_{b, c} \leq 1$ for any $b, c \in \mathcal{C}$ and

$\triangleright k_{i, c} \leq 1$ for any $c \in \mathcal{C}$.

An example of a NE channel allocation corresponding to Theorem 3 is shown in Figure 3(a).

Theorem 3 suggests that players should distribute their radios over the set of available channels. Surprisingly, there exist another type of Nash equilibria in which some players use multiple radios in some channels. We characterize these Nash equilibria in the following theorem.

Theorem 4: Assume that $|\mathcal{N}| \cdot k>|\mathcal{C}|$. Then a channel allocation $S^{*}$ is a NE if the following conditions hold:

$\triangleright \delta_{b, c} \leq 1$ for any $b, c \in \mathcal{C}$ and

$\triangleright$ for any player $i$ that has $k_{i, c} \geq 2$ for $c \in \mathcal{C}, k_{i, c} \leq$ $\frac{r\left(k_{c}-1\right) /\left(k_{c}-1\right)-r\left(k_{c}+1\right) /\left(k_{c}+1\right)}{r\left(k_{c}-1\right) /\left(k_{c}-1\right)-r\left(k_{c}\right) /\left(k_{c}\right)}$ also holds; and

$\triangleright$ for any player $i$ that has $k_{i, b} \geq 2$ and $b \in \mathcal{C}_{\max }$, it is also true that $k_{i, c} \geq k_{i, b}-1, \forall c \in \mathcal{C}_{\text {min }}$

Figure 3(b) presents an example for Theorem 4 assuming that the second (numerical) condition of the theorem holds.

In summary, Theorems 3 and 4 characterize two types of Nash equilibria. In the first type, each player distributes his radios such that he has at most one radio per channel. Intuitively, this results in load balancing. Note, however, the existence of a second type of Nash equilibria, in which some players allocate multiple radios on certain channels. We mention that there could be a small set of other Nash equilibria that are not covered by these theorems, but they exist for very specific conditions on the throughput function $r(\cdot)$. These Nash equilibria can be derived from the proof of Theorem 4.

\section{Efficiency}

In the following, we study the efficiency of the Nash equilibria in the $\mathrm{CA}$ game. In the next theorem, we show that the selfish channel allocation of the players results in an efficient spectrum utilization for the optimal rate function derived in [22] (i.e., if the rate function is independent of the number of radios). 


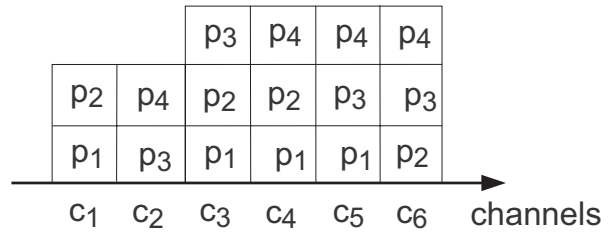

(a)

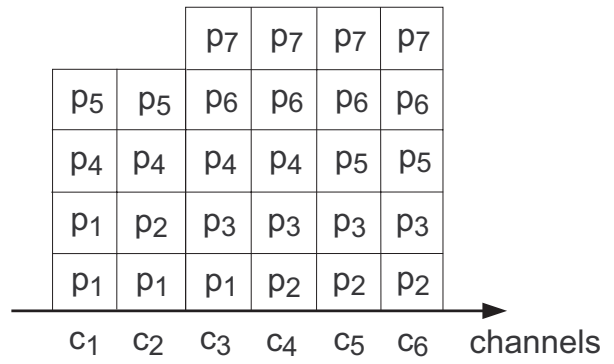

(b)

Fig. 3. Two types of Nash equilibria in the CA game: (a) Each player distributes his radios over the channels (i.e., $k_{i, c} \leq 1, \forall i, \forall c$ ) or (b) certain players use multiple radios on some channels (here player $p_{1}$ uses two radios on channel $c_{1}$ ). In (a) $|\mathcal{C}|=6,|\mathcal{N}|=4$ and $k=4$, whereas in (b) $|\mathcal{C}|=6,|\mathcal{N}|=7$ and $k=4$.

Theorem 5: If the rate function $r(\cdot)$ is independent of $k_{c}$ on any channel $c$, then any NE channel allocation $S^{*}$ is Paretooptimal.

Theorem 5 also means that in the ideal case the price of anarchy is 1 . Note that this result is valid for the ideal CSMA/CA protocol, but it does not hold in the practical case, where $r(\cdot)$ is decreasing with the number of radios on channel $c$. In the practical case, the players might remove some of their radios to decrease the total number of radios on certain channels. If they do this mutually, they could increase each others' payoffs. Clearly, in a Pareto-optimal solution, each channel is used by only one radio.

We can express the efficiency of the Nash equilibria using the concept of the price of anarchy (POA $)^{1}$.

Theorem 6: If $r(\cdot)$ is a decreasing function of $k_{c}$, then the price of anarchy (POA) is given by:

$$
P O A=\frac{r(1)}{\left(k_{c}+1-\frac{|\mathcal{N}| \cdot k}{|\mathcal{C}|}\right)\left(r\left(k_{c}\right)-r\left(k_{c}+1\right)\right)+r\left(k_{c}+1\right)}
$$

where $k_{c}=\left\lfloor\frac{|\mathcal{N}| \cdot k}{|\mathcal{C}|}\right\rfloor$ (i.e., $k_{c}+1=\left\lceil\frac{|\mathcal{N}| \cdot k}{|\mathcal{C}|}\right\rceil$ ).

In practice, the throughput function $r(\cdot)$ is decreasing with the number of users contending for the channel. Nevertheless, it declines rather slowly, notably thanks to the RTS/CTS extension of the basic CSMA/CA protocol. Hence, the first term in the denominator of (6) converges to 0 . As the throughput function converges to a constant function (i.e., being almost independent of the number of radios), the upper bound in (6) converges to 1 . This suggests that the price of anarchy is 1 in theory and it is close to 1 in practice.

\section{CSMA/CA GAME}

In this section, we describe the CSMA/CA game that is played between a group of players that share the same channel (i.e., that have at least one radio on the channel). The CSMA/CA game is played upon completion (convergence) of the previous channel allocation (CA) game (Figure 1). In an attempt to further increase their bandwidth, selfish players may try to tamper with their radio adapters (for example, deliberately reduce the contention window in the case of IEEE 802.11 adapters [3], [27]). In this section, we study

\footnotetext{
${ }^{1}$ Note that all Nash equilibria have the same efficiency and hence the POA definition holds.
}

the stability and efficiency of the group of players (sharing the same channel) where one or several players manipulate their radio adapters; more specifically, selfish players reduce the contention window of their IEEE 802.11 adapters.

As we will show, the CSMA/CA game not only optimizes the bandwidth utilization of every occupied channel, but also provides incentives to the players in the CA game to behave "optimally". By optimally, we mean that a selfish player $i$ will have an incentive to ensure that all the other players learn the exact number of radio adapters on every channel that player $i$ is using; this is an important condition for reaching (nearly) optimal allocations in the CA game. Moreover, the CSMA/CA game ensures per-radio fairness, another important ingredient of the CA game. Due to space constraints, we omit the proofs of our results in this section as well, but they are presented in [28].

\section{A. Preliminaries}

We first introduce the basic assumptions and terminology used in the context of the CSMA/CA game. Recall that a player owns multiple radio adapters, potentially allocated to different radio channels. The same CSMA/CA game is played on each channel in $\mathcal{C}$ between the players who have a radio on that channel. Therefore, in our analysis, we will focus on a single channel $c \in \mathcal{C}$ occupied by multiple radio adapters owned by two or more players. All conclusions drawn from this "single channel" model are applicable to other occupied channels; the channels are indistinguishable in this regard.

We define the set $\mathcal{N}_{c}$ of players that have at least one radio on the observed channel $c \in \mathcal{C}$; note that $\mathcal{N}_{c} \subseteq \mathcal{N}$. In our analysis, we will be dealing exclusively with IEEE 802.11 (in the Distributed Coordination Function (DCF) mode) [27]; however, we note that the analysis carried out in this setting can also be extended to other CSMA/CA based protocols. There can be a number of ways in which players can misbehave with their radio adapters. For example, in violation of the standard protocol, player $i \in \mathcal{N}_{c}$ can initialize the contention window size on his radio adapters to a lower value in order to obtain a higher throughput. We will call this lower value $W_{i, \ell}$, the contention window of player $i$ 's $\ell$-th radio. This mode of cheating is the easiest (and yet highly rewarding), since it does not require changes to be made in the operation of the IEEE 802.11 protocol. We would like to stress that the main 
conclusions of this study are applicable to any other cheating technique.

We will designate those players who selfishly tamper with their adapters as described above, as cheaters against the standard protocol and refer to their set as $\mathcal{I}_{c} \subseteq \mathcal{N}_{c}$. In our model, every cheater $i \in \mathcal{I}_{c}$ seeks to maximize the average throughput $r_{i, c}$ he enjoys on channel $c$. For each cheating player $i \in \mathcal{I}_{c}$, we define the pure-strategy set $\mathcal{S}_{i}$ as follows:

$$
\mathcal{S}_{i}=\underbrace{\left\{1,2, \ldots, W_{\max }, W_{\infty}\right\} \times \ldots \times\left\{1,2, \ldots, W_{\max }, W_{\infty}\right\}}_{k_{i, c}},
$$

where $\times$ is the Cartesian product, $k_{i, c}$ is the number of radio adapters player $i$ uses on channel $c, W_{\max }<\infty$ is a positive integer and the symbol $W_{\infty}$ means that the player $i$ does not transmit at all on channel $c$ using the corresponding adapter, which is equivalent to $W_{i}=\infty$ for that radio adapter. Note that the set $\mathcal{S}_{i}$ is finite. Strategy $s_{i} \in \mathcal{S}_{i}$ of each player $i \in \mathcal{I}_{c}$ in the CSMA/CA game consist in choosing the contention window values $s_{i}=\left(W_{i, 1}, W_{i, 2}, \ldots, W_{i, k_{i, c}}\right)$ such that player $i$ 's throughput $r_{i, c}$ is maximized on each channel $c$.

We modified the theoretical model of Bianchi [22] to derive the throughput enjoyed by the cheaters tampering with the 802.11 protocol backoff parameter. A detailed model and the resulting derivation is presented in [28]. For easier mathematical treatment, we use the channel access probabilities $\tau_{i, \ell}=\frac{1}{W_{i, \ell}+1}\left(\ell \in\left\{1, \ldots, k_{i, c}\right\}\right)$ instead of working directly with contention windows $W_{i, \ell}\left(\ell \in\left\{1, \ldots, k_{i, c}\right\}\right)$. Let $\tau$ denote the channel access probabilities of non-cheating nodes. Let us also denote with $K_{i, c}$ the set of radio adapters that player $i$ uses on channel $c$, that is, $K_{i, c}=\left\{1,2, \ldots, k_{i, c}\right\}$. Then, we can express the (average) throughput $r_{i, c}$ of a cheater $i \in \mathcal{I}_{c}$ on channel $c$ as:

$$
r_{i, c}=\sum_{\ell \in K_{i, c}} r_{i, c, \ell},
$$

where $r_{i, c, \ell}$ is the average throughput of player $i$ 's $\ell$-th radio adapter used on channel $c$ and it is given by expression (7).

In expression (7), $L$ represents the average packet length, $T^{s}$ is the average time needed to transmit a packet of size $L$ (including the inter-frame spacing periods), $T^{\text {id }}$ is the duration of the idle period (a single slot) and $T^{c}$ is the average time spent in the collision. Moreover, we used two substitutions $p_{-i}$ and $q_{-i}$ given by expressions (8) and (9), respectively.
Note here, that the only parameter that a cheating node $i$ has a control over is its own $\tau_{i, \ell}$ by manipulating $W_{i, \ell}$.

We denote the game as defined in this subsection by $G_{\mathrm{CSMA} / \mathrm{CA}}=\left\langle\mathcal{I}_{c},\left(\mathcal{S}_{i}\right)_{i \in \mathcal{I}_{c}},\left(r_{i, c}\right)_{i \in \mathcal{I}_{c}}\right\rangle$ and call it a static CSMA/CA game played on channel $c \in \mathcal{C}$.

\section{B. Static CSMA/CA Game: Inefficient, Unfair and Nonessen- tial}

In this subsection, we will characterize possible Nash equilibria of the static CSMA/CA game $G_{\mathrm{CSMA} / \mathrm{CA}}$ played on the observed channel $c$. We first state the following straightforward lemma.

Lemma 7: For any strategy profile $s^{*}=\left(s_{1}^{*}, \ldots, s_{\left|\mathcal{I}_{c}\right|}^{*}\right)$ that constitutes a Nash equilibrium in $G_{\mathrm{CSMA} / \mathrm{CA}}$, there exists a cheating player $i \in \mathcal{I}_{c}$ with strategy $s_{i}^{*}=$ $\left(W_{i, 1}, W_{i, 2}, \ldots, W_{i, k_{i, c}}\right) \in S_{i}$ such that at least one $W_{i, \ell}$, $\ell=1, \ldots, k_{i, c}$, is set to 1 .

The intuition of the lemma is that if a cheater $i$ decreases the contention window $W_{i, \ell}$ of her device $\ell$, then $r_{i, c, \ell}$ throughput either increases or remains the same. Based on this reasoning, at least one cheater $i$ is going to use the minimum contention window 1 for one of her adapters. Lemma 7 gives a necessary condition for a strategy profile to be a Nash equilibrium. In [28] we show that Lemma 7 provides also a sufficient condition. In other words, any strategy profile $s$ such that at least one contention window $W_{i, \ell}, \forall i \in \mathcal{I}_{c}$ and $\forall \ell=1, \ldots, k_{i, c}$, equals 1 is a Nash equilibrium. From this we can easily count that the static CSMA/CA game admits $\left(W_{\max }+1\right)^{\sum_{i \in \mathcal{I}_{c}} k_{i, c}}-W_{\max }^{\sum_{i \in \mathcal{I}_{c}} k_{i, c}}$ Nash equilibria. It is more interesting, however, to observe that the equilibria can be classified into two types. In the 1 st type, there is only one player $i \in \mathcal{I}_{c}$ who sets exactly one of the contention windows $W_{i, \ell}, \ell=1, \ldots, k_{i, c}$, to 1 and receives a non-null throughput $r_{i, c}>0$, and $r_{k, c}=0$ for all players $k \in \mathcal{I}_{c} \backslash\{i\}$. In the Nash equilibria of the 2 nd type, more than one player $i \in \mathcal{I}_{c}$ set at least one of their contention $W_{i, \ell}, \ell=1, \ldots, k_{i, c}$, to 1 , in which case $r_{i, c}=0$, for all players $i \in \mathcal{I}_{c}$. This 2 nd type of equilibria corresponds to the well-known tragedy of the commons in game theory, because finally no player obtains any useful throughput.

It is generally believed that for noncooperative channel access games the tragedy of the commons (2nd type) is the most likely equilibrium [24]. However, we have seen that

$$
\begin{aligned}
& r_{i, c, \ell}=\frac{\tau_{i, \ell} \cdot p_{-i, \ell} \cdot L}{\tau_{i, \ell} \cdot\left(p_{-i, \ell}\left(T^{s}-T^{i d}\right)-q_{-i, \ell}\left(T^{s}-T^{c}\right)\right)+\left(1-p_{-i, \ell}-q_{-i, \ell}\right) T^{c}+q_{-i, \ell} T^{s}+p_{-i, \ell} T^{i d}} \\
& p_{-i, \ell}=(1-\tau)^{\left|\mathcal{N}_{c}\right|-\left|\mathcal{I}_{c}\right|} \times \prod_{\substack{j \in \mathcal{I}_{c} \\
k \in \widehat{K}_{j, c}}}\left(1-\tau_{j, k}\right), \text { where } \widehat{K}_{j, c} \stackrel{\text { def }}{=} \begin{cases}K_{j, c}, & j \neq i \\
K_{j, c} \backslash\{\ell\}, & j=i .\end{cases} \\
& q_{-i, \ell}=(1-\tau)^{\left|\mathcal{N}_{c}\right|-\left|\mathcal{I}_{c}\right|} \times \sum_{\substack{j \in \mathcal{I}_{c} \\
k \in \widehat{K}_{j, c}}} \tau_{j, k} \prod_{\substack{m \in \mathcal{I}_{c} \\
n \in \widehat{K}_{m, c}}}\left(1-\tau_{m, n}\right) \text { where } \widehat{K}_{m, c} \stackrel{\text { def }}{=} \begin{cases}K_{m, c}, & m \neq j, m \neq i ; \\
K_{m, c} \backslash\{k\}, & m=j, m \neq i ; \\
K_{m, c} \backslash\{\ell\}, & m \neq j, m=i ; \\
K_{m, c} \backslash\{k, \ell\}, & m=j, m=i .\end{cases}
\end{aligned}
$$


there exist another family of Nash equilibria in $G_{\mathrm{CSMA} / \mathrm{CA}}$. It can be shown that the tragedy of the commons equilibria are nonessential (non robust) in the game $G_{\mathrm{CSMA} / \mathrm{CA}}$, which further implies that the game $G_{\mathrm{CSMA} / \mathrm{CA}}$ is nonessential [24] $]^{2}$. Therefore, we look for an alternative solution to $G_{\mathrm{CSMA} / \mathrm{CA}}$ by allowing the players to agree on the strategies they will use.

\section{Unique, Per-Radio Fair and Pareto-Optimal Solution}

Intuitively, a desirable solution for the CSMA/CA game should exhibit the following three properties: (i) uniqueness - this is to avoid uncertainties with respect to what solution each player should choose, (ii) per-radio fairness - since, in general, a player doesn't know how many and what adapters belong to another player, (iii) Pareto optimality - the solution should result in a Pareto optimal allocation of the available bandwidth.

In order to derive such a solution, we use the Nash Bargaining Framework (NBF) from the theory of cooperative games [28], [29]. From the NBF, we know that we can obtain a unique, fair (per-radio) and Pareto-optimal solution by solving the following maximization problem:

$$
\begin{array}{cc}
\text { maximize } & \prod_{i \in \mathcal{I}_{c}} \prod_{\ell=1}^{k_{i, c}} r_{i, c, \ell}\left(s_{1}, s_{2}, \ldots, s_{\left|\mathcal{I}_{c}\right|}\right) \\
\text { subject to } & s_{i} \in S_{i}, \forall i \in \mathcal{I}_{c}
\end{array}
$$

where $r_{i, c, \ell}\left(s_{1}, s_{2}, \ldots, s_{\left|\mathcal{I}_{c}\right|}\right)$ represents the throughput of player $i$ 's $\ell$-th radio adapter used on channel $c$. Note that the above optimization problem is discrete. In [28], we proved that a continuous version of this problem admits a unique solution which is also per-radio fair and Pareto-optimal. We obtain a a continuous version by relaxing the integrality constraints on the variables (contention windows) $W_{i, \ell}$. Here we only state the final result.

Theorem 8: A continuous version of the optimization problem (10) admits a unique solution $\tau_{i, \ell}=\tau^{*}, \forall i \in \mathcal{I}_{c}$ and $\ell=1, \ldots, k_{i, c}$, with $\tau^{*} \in(0,1)$ (for finite number of players). Clearly, the unique solution is also Pareto-optimal and perradio fair. Note that $\tau_{i, \ell}=\tau^{*}, \forall i \in \mathcal{I}_{c}$ and $\ell=1, \ldots, k_{i, c}$, implies $W_{i, \ell}=\frac{1}{\tau^{*}}-1, \forall i \in \mathcal{I}_{c}$ and $\ell=1, \ldots, k_{i, c}$.

In order to show the existence of an optimal integer value of the contention window, in Figure 4 we plot the average aggregated throughput obtained by 10 cheating players, all of which use the same contention window size and have one adapter on the shared channel. Note that in the simulations we take the players that respect IEEE 802.11 protocol into account; they, however, do not affect the qualitative conclusions of the analytical treatment in this section. From this figure we can see that there exists a unique joint contention window size $W^{*}$ maximizing the system throughput, which is consistent with the conclusion of Theorem 8 .

We conclude that the optimal solution $W^{*}$ to the optimization problem (10) exhibits all the properties of a desirable point of operation in the CSMA/CA game $G_{\mathrm{CSMA} / \mathrm{CA}}$. In our context, this is significant as the Pareto-optimal solution is not a Nash equilibrium point and as such might not be stable.

\footnotetext{
${ }^{2}$ Informally, a game is non-essential if the Nash equilibria are non-robust to small perturbations in the payoff structure of the game. Note that the small change can result from inability of the game modeler to specify payoff functions that are perfectly correct.
}

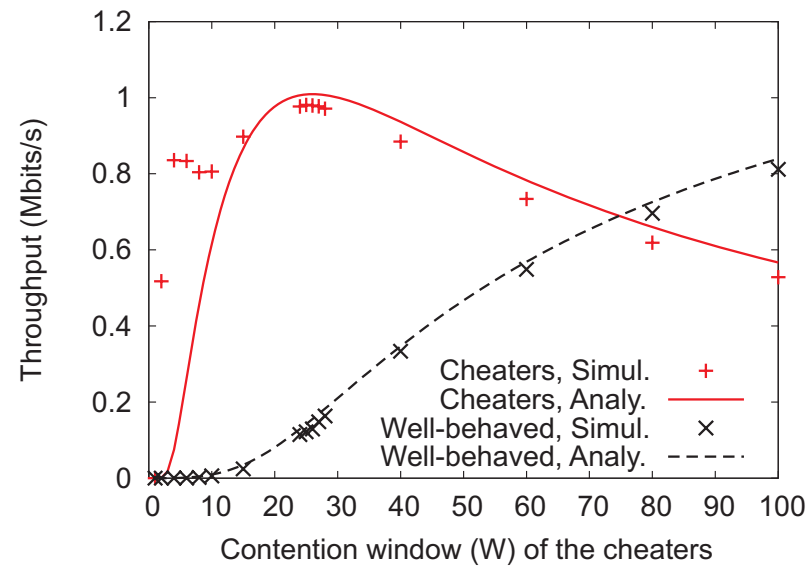

Fig. 4. Throughput vs. contention window size of the cheating players: there are 20 nodes on the observed channels, out of which 10 cheating players, i.e., $\left|\mathcal{I}_{c}\right|=10$, each player has exactly one adapter on this channel.

Therefore, in the following subsection, we look at how to make the unique, per-radio fair and Pareto-optimal point $W^{*}$ a Nash equilibrium point.

\section{Penalizing Mechanism: Towards a Unique and Pareto- Optimal Nash Equilibrium}

Having determined the desirable point of operation, we now intend to devise a strategy that allows the players to converge to this point. At the core of this strategy is the penalty mechanism, by which one player can penalize severe deviations of another player.

Let us consider two arbitrary players $i$ and $j$ from the set $\mathcal{I}_{c}$ and their arbitrary radio adapters $m$ and $n$ used on the common channel $c$. Let us assume that player $i$ calculates the penalty $p_{j, c, n}$ to be inflicted on player $j$ 's $n$-th radio adapter as follows

$p_{j, c, n}(s)= \begin{cases}r_{j, c, n}(s)-r_{i, c, m}(s), & \text { if } r_{j, c, n}(s)>r_{i, c, m}(s) \\ 0, & \text { otherwise }\end{cases}$

Then the throughput of player $j$ 's $n$-th adapter is $r_{j, c, n}(s)-$ $p_{j, c, n}(s)=r_{i, c, m}(s)$. Thus, the two adapters $m$ and $n$ receive the same throughputs. Inspired by the penalty functions (11), we have designed a simple penalizing scheme, in which the packets of the noncooperative player's adapter are selectively jammed for a short duration of time, $T^{j a m}$, by the other players using the channel. By the "noncooperative adapter" we mean the adapter that deviates from the given equilibrium point (e.g., the Pareto-optimal point). Suppose that a player $i \in \mathcal{I}_{c}$ detects the presence of a noncooperative player $j \in \mathcal{I}_{c}$. Thereafter, if the player $i$ listens to a transmitted packet corresponding to the player $j$ 's noncooperative adapter, it switches to transmission mode and jams enough bits so that the packet cannot be properly recovered at the receiver.

Let the throughput obtained by the two adapters $m$ and $n$ of players $i$ and $j$ over the last observation window, $T^{\text {obs }}$, be $r_{i, c, m}$ and $r_{j, c, n}$, respectively, where $r_{i, c, m}>r_{j, c, n}$. As we presented above, the penalty function (11) aims at making the throughputs received by adapters $m$ and $n$ equal. We denote with $r_{i, c, \ell}(t)$ the instantaneous throughput of adapter $\ell$. The average throughput received by the adapter $m$ and $n$ should 


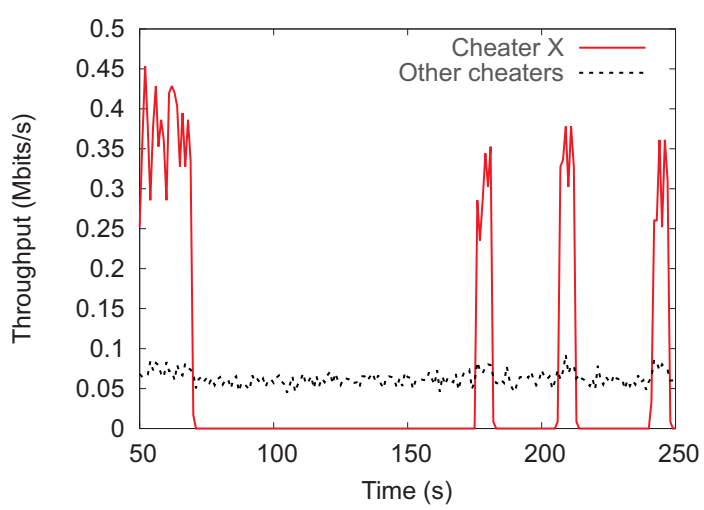

(a)

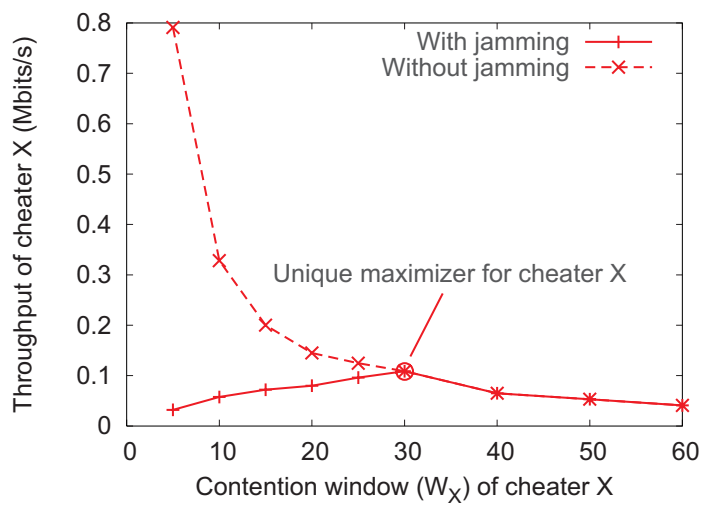

(b)

Fig. 5. Realization of the penalty through selective jamming: (a) Throughputs (payoffs) obtained by the cheaters over time in the presence of the noncooperative cheater $X$ and selective jamming mechanism; (b) Unilateral deviation by the cheater $X$ with and without the penalty mechanism.

be the same over the total time duration of $T^{o b s}+T^{j a m}$, that is,

$$
\begin{aligned}
& \frac{1}{T^{o b s}+T^{j a m}} \int_{t}^{t+T^{o b s}+T^{j a m}} r_{i, c, m}(t) d t= \\
= & \frac{1}{T^{o b s}+T^{j a m}} \int_{t}^{t+T^{o b s}} r_{j, c, n}(t) d t,
\end{aligned}
$$

where we have used the fact that the player $i$ jams player $j$ 's adapter during the period $T^{j a m}$. Let us denote the average throughput over a time period $P$ starting at time instant $t$ by $\bar{r}(t, P)$, that is, $\bar{r}(t, P)=\frac{1}{P} \int_{t}^{t+P} r(t) d t$. Then, from the expression (12) we obtain

$$
T^{j a m}=T^{o b s} \frac{\bar{r}_{j, c, n}\left(t, T^{o b s}\right)-\bar{r}_{i, c, m}\left(t, T^{o b s}\right)}{\bar{r}_{i, c, m}\left(t+T^{o b s}, T^{j a m}\right)} .
$$

We have implemented the jamming mechanism in $n s-2$. The simulation setup is the previous section with 20 nodes using the channel $c$ and 10 cheating players (i.e., $\left|\mathcal{I}_{c}\right|=10$ ); every player has only one adapter on channel $c$. We randomly pick up a cheating player, designated as cheater $X$, and fix his contention window size to be 10 . The contention window size for all the other cheaters in the system is fixed to the point 30 . We use an observation window size, $T^{\text {obs }}$, of 20 seconds. Cheater $X$ is detected by the other cheaters in the network (e.g., using the mechanisms described in [27]) and is penalized for his deviation.

In Figure 5(a), we plot the throughput obtained by the cheaters in the system over time, with and without the penalizing scheme. As can be observed from Figure 5(a), cheater $X$ is detected and is penalized for his deviation. When penalized, the cheater $X$ 's throughput drops to zero. Observe from this figure the dependency of the period $T^{j a m}$ on the observation period $T^{o b s}$; for better system efficiency, $T^{o b s}$ should be kept short (much shorter than 20 seconds as used in our simulations).

Figure 5(b) plots the average throughput obtained by cheater $X$, when it unilaterally deviates from the given equilibrium point 30 . The results are averaged over a duration of 1000 seconds. As can be observed from Figure 5(b), after the introduction of the detection and penalizing mechanism, cheater $X$ achieves maximum throughput by operating at the given equilibrium point where all cheater set their contention window to 30 . Thus, any unilateral deviation from this point brings less payoff to the cheater $X$. Therefore, by definition, this equilibrium point is a Nash equilibrium - unilateral deviation is not profitable.

We conclude that by using penalty function as given in (11), we can make an arbitrary value of the contention window a Nash equilibrium point, including a value that is obtained as the desirable Pareto-optimal solution of the optimization problem (10) (Section VI-C).

\section{CONVERGEncE TO THE EFFICIENT NASH EQUILIBRIA}

In this section, we propose one distributed protocol in each game to achieve the identified Pareto-optimal Nash equilibria. We also study the convergence properties of these protocols.

\section{A. Convergence in the CA Game}

In Section V, we presented a set of theoretical results to identify Nash equilibria in the CA game and we showed that they are efficient. We assumed that there is perfect information [24], [26] available for the players about the set of radios on each channel. In practice, the devices only know the number of radios on the channels on which they have a radio themselves.

In this subsection, we propose a distributed convergence algorithm to show that the previously identified Nash equilibria are achievable even in the case of imperfect information. We define a round-based distributed algorithm that works as follows [26]. First, we assume that there exists a random radio assignment of the players over the channels. For simplicity, we exclude the Nash equilibria that correspond to Theorem 4. This means that we assume that no player allocates more than one device on any channel. After the initial channel assignment, each player evaluates the number of radios (which defines the approximate length of the round) on each of the channels on the channels he knows (recall that we denote this set by $\mathcal{C}_{i}$ ) and decides with probability $\rho$ to improve his total throughput by reorganizing his radios. This probability is necessary to avoid continuous reallocation of the radios if the players change in the same round. We denote the average number of devices on the channels in $\mathcal{C}_{i}$ by $K_{i}$ (note that $K_{i}$ is not necessarily an integer). For each channel $b \in \mathcal{C}_{i}$ with $k_{b}-K_{i} \geq 1$, player $i$ 
moves his radio to another channel $c \notin \mathcal{C}_{i}$. The probability to choose a channel $c \notin \mathcal{C}_{i}$ is $\frac{1}{\mathcal{C} \mid \backslash \mathcal{C}_{i}}$. This is the first property of the algorithm with imperfect information.

We can show that the above procedure reaches a stable state. Unfortunately, the available local information might be insufficient for the players to determine if the achieved stable state is a Nash equilibrium (in rare cases, the stable state is not a Nash equilibrium). Hence, the players use a second probability $\epsilon$ to change even if they believe that a stable state is reached (i.e., when $0<k_{b}-K_{i}<1$ for each channel $b \in \mathcal{C}_{i}$ ). This second property allows us to resolve the inefficient stability

states, but at the same time, it will also cause the instability of the Nash equilibria. Note, however, that the amount of instability can be tuned using the parameter $\epsilon$.

We calculate the average number of radios per channel as $K=\frac{|\mathcal{N}| \cdot k}{|\mathcal{C}|}$. We can compare the utilization of every channel $x$ to the average to achieve the total balance of the channel allocation $S$ :

Definition 4: (Balance:) The balance $\beta$ of a channel allocation $S$ is defined as the sum $\beta(S)=\sum_{c \in \mathcal{C}}\left|k_{c}-K\right|$.

Let us first highlight the best and worst case in terms of the desired load-balancing for this algorithm. The best case is one of the NE channel allocations. The worst case, called the unbalanced $(U B)$ channel allocation, is characterized by the fact that there exist $k$ channels where each of the players have a radio, whereas the rest of the channels have no radios at all.

The notion of balance allows us to define the efficiency of a given channel allocation as a proportion between the worst case and the best case channel allocations.

Definition 5: (Efficiency:) The efficiency $\phi$ of a channel allocation $S$ is defined as $\phi(S)=\frac{\beta\left(S_{\mathrm{UB}}\right)-\beta(S)}{\beta\left(S_{\mathrm{UB}}\right)-\beta\left(S_{\mathrm{NE}}\right)}$.

Let us emphasize that for any channel allocation $S$, it is true that $0 \leq \phi(S) \leq 1$. Furthermore, $\phi\left(S_{\mathrm{NE}}\right)=1$ and $\phi\left(S_{\mathrm{UB}}\right)=$ 0 as desired by this measure.

Now we present an example run for our distributed algorithm with imperfect information in Figure 6(a) for 5000s. Here, we assume that the duration of one round (i.e., the time needed to learn the number of radios on a given channel) is equal to $100 \mathrm{~ms}$. One can notice that the algorithm quickly reaches the NE state, i.e., the efficiency reaches one. Simulation results show that this convergence time is in the order of 0.5 seconds. Also, one can observe that the players sometimes leave the NE state due to the second property, but they quickly return to it. Suppose that the total available throughput per channel is $r\left(k_{c}\right)=54 \mathrm{Mbps}$, for any $k_{c}$. Figure 6(b) presents a snapshot of the total payoff of the players in the first NE reached in the previous simulation. One can observe that the total throughput is very similar for the users, hence we conclude that our algorithm converges to load-balancing, fair channel allocations.

\section{B. Convergence in the CSMA/CA Game}

In this subsection, we give a simple algorithm that is based on the penalty function (11) proposed in Section VI-D and that leads the players (their adapters on the observed channel) to a unique Pareto-optimal Nash equilibrium. The main idea is that one player $i$ (his adapter $m$ ) acts as a coordinator on the observed channel by inflicting penalties on the radios of other players who receive a higher throughput. The equilibrium coordination procedure is an interplay between two simple algorithms.

First, the coordinating adapter $m$ chooses some initial contention window value $W_{i, m}=W_{0}>1$ and begins to penalize the other players's adapters (on the same channel) with $r_{j, c, n}(s)>r_{i, c, m}(s), j \neq i$. The other players (affected adapters) act selfishly and run the adaptation algorithm that maximizes $r_{i, c, m}(s)$ (due to the penalty function (11)). This algorithm simply attempts to equalize the other adapters' contention windows with the coordinator's contention window $W_{i, m}=W_{0}$; recall from Figure $5(\mathrm{~b})$ that this value maximizes the throughput of each adapter. This stage lasts until all the adapters that belong to the cheating players on the observed channel, stabilize at a stage Nash equilibrium point $W_{i^{\prime}, \ell}=W_{0}, i^{\prime} \in \mathcal{I}_{c}$ and $\ell=1, \ldots, k_{i, c}$.

After spending some finite time on this point (to let all the adapters to learn that the system has reached an equilibrium point), the coordinator further updates his contention window to value $W_{1}>W_{0}$ and again begins to penalize the other players's adapters (on the same channel) with $r_{j, c, n}(s)>$ $r_{i, c, m}(s), j \neq i$. In turn, the other players selfishly adjust the contention windows of their adapter in order to maximize their throughputs. At this stage, the coordinator compares the throughput $r_{i, c, m}\left(W_{0}\right)$ received at the previous equilibrium point $W_{0}$ and the throughput $r_{i, c, m}\left(W_{1}\right)$ at the current equilibrium point $W_{1}$. If the difference is $\left|r_{i, c, m}\left(W_{0}\right)-r_{i, c, m}\left(W_{1}\right)\right|$ is smaller than some small value $\Delta$, the coordinator stops increasing his contention window. The rationale here is that the coordinator pulls all the other cheating adapters up to the left side of the aggregate throughput curve shown in Figure 4, until they all reach the maximum point, the Pareto-optimal Nash equilibrium (due to the penalty function, no player had an incentive to unilaterally deviate). It is important to emphasize that there can be multiple groups of simultaneously coordinating adapters.

We have implemented the proposed coordination procedure in $n s-2$. The simulation setup consists of 20 players out of which 7 cheaters $\left(\left|\mathcal{I}_{c}\right|=7\right)$, all using the same channel. Each player has only one radio adapter. The cheating players initially set their contention windows to $5\left(W_{i, \ell}=5, i \in \mathcal{I}_{c}\right.$, $\left.\ell=1, \ldots, k_{i, c}\right)$. The cheaters continue their search for the Pareto-optimal point $W^{*}$ only if they see an increase of $10 \%$ or more in their throughput from the last stable point of operation.

Figure 7(a) plots the sample evolution of the contention window for 2 cheating adapters, $X$ and $Y$, in the system. Note that all of the cheaters follow a similar pattern and eventually converge to a window size of 20 . We are unable to show their evolution in the same plot as it simply generates overlapping lines. Note also that the convergence time is relatively short, around 80 seconds for 7 cheaters (from $t_{\text {start }} \approx 160$ to $t_{\text {end }} \approx 240$; in these simulations we used a warm-up period of around 160 seconds). It is important to observe that 80 seconds incorporate the time for both the detection and the penalization of potential deviations from the current equilibrium point. What is more, the cheating adapters 


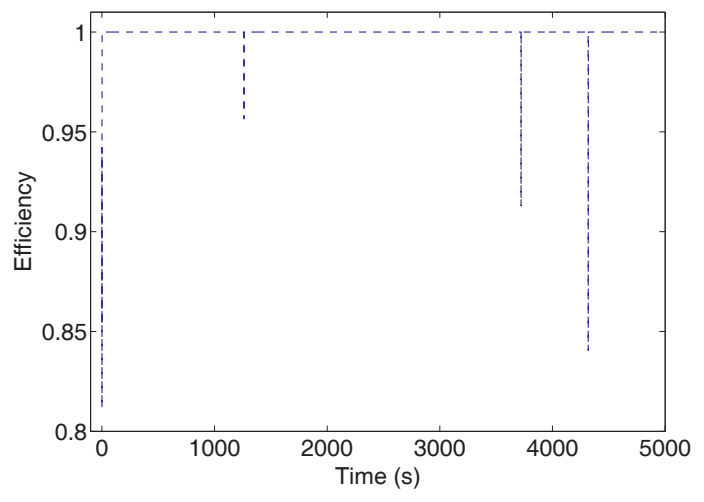

(a)

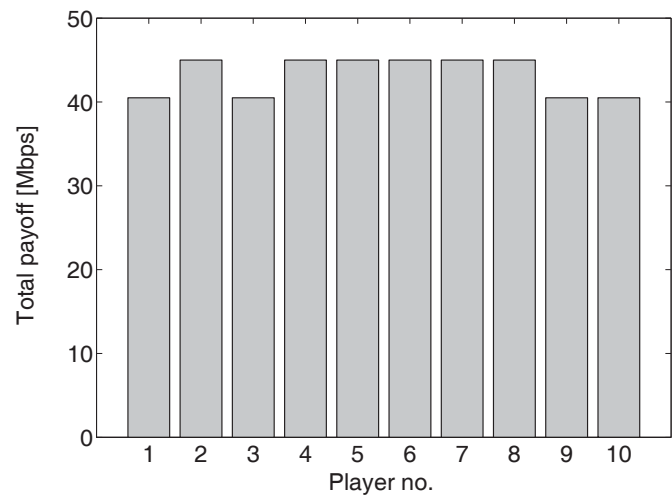

(b)

Fig. 6. Example simulation run: (a) The efficiency and (b) the total payoff of the players in the first NE. The parameter values are $|\mathcal{C}|=8,|\mathcal{N}|=10$, $k=3, \epsilon=10^{-5}$ and $W=15$.

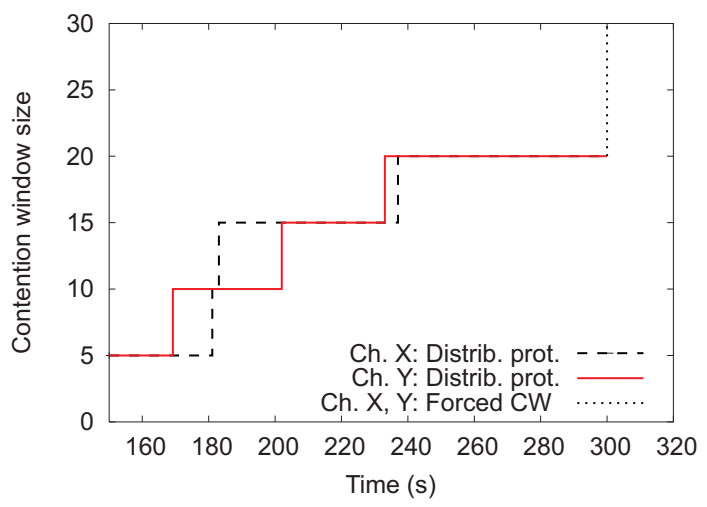

(a)

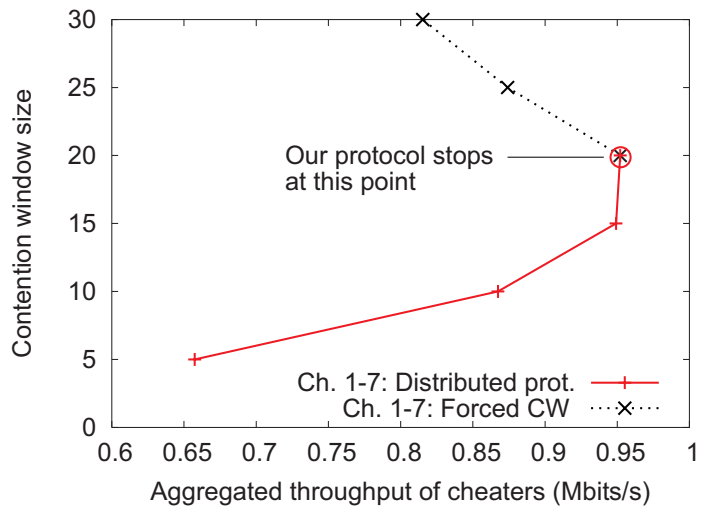

(b)

Fig. 7. Performance of the distributed coordination protocol, with 20 players and $\left|\mathcal{I}_{c}\right|=7$ (the axes in (b) are swapped for the convenience of matching them with (a)): (a) Evolution of the contention windows; (b) Contention window vs. Average throughput.

receive positive throughputs (payoffs) during the coordination procedure.

Figure 7(b) plots the average throughput obtained by the cheaters at different contention window sizes. As can be seen from Figure 7(b), the throughput is maximized at $W_{i, \ell}=20$, $\forall i \in \mathcal{I}_{c}$ and $\forall \ell=1, \ldots, k_{i, c}$. For completeness, we obtain the "dotted" curves in Figure 7 by deliberately forcing the cheaters to go beyond $W_{i, \ell}=20$.

\section{Mutual Reinforcement between the CA and the CSMA/CA Games}

As we have seen in Section VI, a selfish player $i$ might manipulate the channel access of his radios by choosing a very small backoff window value in the CSMA/CA protocol on any channel $c$ to obtain the total achievable throughput while the other radios receive zero throughput. Let us call this behavior the fully selfish behavior in the CSMA/CA game. In Theorem 9, we show that this behavior is discouraged by the CA game: If a fully selfish player $i$ seizes the access to channel $c$, then other players believe that $i$ is the only player on that channel. This attracts them to channel $c$ in the CA game.

Theorem 9: If the players expect a fair channel allocation on each channel $c$ and choose their channel allocation strategies accordingly, then it is not beneficial for any player to be fully selfish on any channel.
Proof: Assume that player $i$ is fully selfish on channel $c$ with one pair of radios (i.e., he manipulates his backoff window such that he is the only one who gets access to the channel). For simplicity, we assume that player $i$ has only one radio on channel $c$, but the proof is similar if he uses several radios. In this case, the other players falsely observe that there is only one radio on channel $c$. Hence, they place their radios on this channel in the CA game.

In the following CSMA/CA game, the other players detect quickly that player $i$ is fully selfish and hence they punish him using the penalty mechanism presented in Section VI-D. Hence, player $i$ obtains no useful throughput in the CSMA/CA game and the period after that. We can express his payoff if he is fully selfish as $r_{i, c}^{f s}=0$. Whereas, if he is not, he obtains his fair share of the throughput $r_{i, c}^{n f s}=\frac{1}{k_{c}} \cdot r\left(k_{c}\right)$. We know that $k_{c}>0$ and $r\left(k_{c}\right)>0$ and thus player $i$ is always better off being not fully selfish.

Note that the fully selfish player achieves some throughput in the CA game, but, as we have seen previously in this section, the CSMA/CA game is longer than the CA game. This means that the initial gain from being fully selfish is outweighed by the loss he suffers later.

We also have to mention that Theorem 9 does not exclude selfishness that has smaller impact than the fully selfish behavior. Note, however, that the penalty mechanism presented in 
Section VI-D detects more subtle manipulation of the backoff windows as well. But, the detection time might be longer in this case.

\section{CONCLUSION}

In this paper, we have considered the problem of competitive medium access in cognitive radio networks. We modeled the problem as a two-tier non-cooperative medium access game composed of a channel allocation (CA) and a multiple access (CSMA/CA) sub-games. In the CA game, we have shown that the price of anarchy is close to 1 , meaning that Nash equilibria are socially desirable, load balancing channel allocations. We have demonstrated that the CSMA/CA game admits a unique, fair and Pareto-optimal Nash equilibrium. In both cases, we proposed simple algorithms to achieve the efficient Nash equilibria.

In terms of future work, we will extend our game model to study selfish medium access in general topology networks. We will also consider the effect of more realistic channel models and the presence of primary users on the Nash equilibrium outcomes.

\section{REFERENCES}

[1] S. Haykin, "Cognitive radio: brain-empowered wireless communications," IEEE J. Select. Areas Commun., vol. 23, no. 2, pp. 201-220, Feb. 2005.

[2] J. M. III, "Cognitive radio for flexible mobile multimedia communications," ACM Mobile Networks Applications, vol. 6, no. 5, pp. 435-441, 2001.

[3] M. Cagalj, S. Ganeriwal, I. Aad, and J.-P. Hubaux, "On selfish behavior in CSMA/CA networks," in Proc. IEEE Conf. Computer Commun. (INFOCOM '05), Miami, USA, Mar. 2005.

[4] M. Félegyházi, M. Cagalj, S. Saeedi, and J.-P. Hubaux, "Non-cooperative multi-radio channel allocation in wireless networks," in Proc. IEEE Conf. Computer Commun. (INFOCOM '07), Anchorage, USA, May 2007.

[5] P. Mähönen, "Cognitive trends in making: future of networks," in Proc. PIMRC'04, Barcelona, Spain, Sept. 2004.

[6] F. Wang, M. Krunz, and S. Cui, "Price-based spectrum management in cognitive radio networks," IEEE J. Select. Topics Signal Processing, vol. 2, no. 1, pp. 74-87, 2008.

[7] C. Zou, T. Jin, C. Chigan, and Z. Tian, "QoS-aware distributed spectrum sharing for heterogeneous wireless cognitive networks," Elsevier J. Computer Networks, special issue on cognitive wireless networks, vol. 52, no. 4, pp. 864-878, 2008.

[8] I. F. Akyildiz, X. Wang, and W. Wang, "Wireless mesh networks: a survey," Computer Networks, vol. 47, pp. 445-487, Mar. 2005.

[9] A. Adya, P. Bahl, J. Padhye, A. Wolman, and L. Zhou, "A multiradio unification protocol for IEEE 802.11 wireless networks," in Proc. Broadnets'04, San Jose, USA, Oct. 2004, pp. 344-354.

[10] M. Alicherry, R. Bhatia, and L. E. Li, "Joint channel assignment and routing for throughput optimization in multi-radio wireless mesh networks," in Proc. ACM/IEEE International Conf. Mobile Computing Networking (Mobicom'05), Cologne, Germany, Aug. 2002, pp. 58-72.

[11] A. Raniwala and T.-C. Chiueh, "Architecture and algorithms for an IEEE 802.11-based multi-channel wireless mesh network," in Proc. IEEE Conf. Computer Commun. (INFOCOM '05), Miami, USA, Mar. 2005.

[12] A. Mishra, S. Banerjee, and W. Arbaugh, "Weighted coloring based channel assignment for WLANs," Mobile Computing Commun. Review $(M C 2 R)$, vol. 9, no. 3, 2005.

[13] H. Zheng and L. Cao, "Device-centric spectrum management," in Proc. IEEE DySPAN'05), Baltimore, USA, Nov. 2005.

[14] J. Konorski, "Multiple access in ad-hoc wireless LANs with noncooperative stations," in Proc. Networking 2002, 2002, pp. 1141-1146.

[15] A. B. MacKenzie and S. B. Wicker, "Stability of multipacket slotted Aloha with selfish users and perfect information," in Proc. IEEE Conf. Computer Commun. (INFOCOM '03), San Francisco, USA, Mar. 2003.

[16] M. M. Halldórsson, J. Y. Halpern, L. E. Li, and V. S. Mirrokni, "On spectrum sharing games," in Proc. 23rd Annual ACM Symposium Principles Distributed Computing (PODC 2004), St. John's, Newfoundland, Canada, July 2004, pp. 107-114.
[17] N. Nie and C. Comaniciu, "Adaptive channel allocation spectrum etiquette for cognitive radio networks," in Proc. IEEE Dynamic Spectrum Access Networks (DySPAN'05), Baltimore, USA, Nov. 2005.

[18] J. O. Neel, J. H. Reed, and R. Gilles, "Convergence of cognitive radio networks," in Proc. Wireless Commun. Networking Conf. (WCNC'04), Atlanta, USA, Mar. 2004.

[19] L. Cao and H. Zheng, "Distributed spectrum allocation via local bargaining," in Proc. IEEE SECON'05, Santa Clara, California, USA, Sept. 2005.

[20] M. Schwartz, Mobile Wireless Commun. Cambridge Univ. Press, 2005.

[21] T. Nandagopal, T. Kim, X. Gao, and V. Bhargavan, "Achieving MAC layer fairness in wireless packet networks," in Proc. ACM MobiCom'00, Boston, USA, 2000.

[22] G. Bianchi, "Performance analysis of the IEEE 802.11 distributed coordination function," IEEE J. Select. Areas Commun., vol. 18, no. 3, Mar. 2000.

[23] M. Félegyházi and J.-P. Hubaux, "Game theory in wireless networks: a tutorial," EPFL, Tech. Rep. LCA-REPORT-2006-002, Feb. 2006.

[24] D. Fudenberg and J. Tirole, Game Theory. MIT Press, 1991.

[25] E. Koutsoupias and C. Papadimitriou, "Worst-case equilibria," in Proc. 16th Annual Symposium Theoretical Aspects Computer Science (STACS'99), Trier, Germany, Mar. 1999.

[26] M. Félegyházi, "Non-cooperative behavior in wireless networks," Ph.D. dissertation, EPFL-Switzerland, Apr. 2007, nr. 3791.

[27] M. Raya, I. Aad, J.-P. Hubaux, and A. E. Fawal, "DOMINO: detecting MAC layer greedy behavior in IEEE 802.11 hotspots," IEEE Trans. Mobile Computing, vol. 5, no. 12, 2006.

[28] M. Cagalj, "Thwarting selfish and malicious behavior in wireless networks," Ph.D. dissertation, EPFL-Switzerland, Feb. 2006, nr. 3449.

[29] M. J. Osborne and A. Rubinstein, A Course in Game Theory. Cambridge, MA: The MIT Press, 1994.

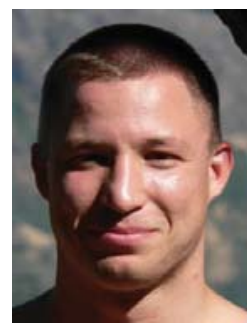

Márk Félegyházi received the M.Sc. degree in Electrical Engineering from the Budapest University of Technology and Economics (BME), Hungary in 2001, and earned the Ph.D. degree from EPFL (Swiss Federal Institute of Technology - Lausanne), Switzerland in 2007. From 1999 to 2001, he was a student member of the Traffic Lab at Ericsson Research, Hungary working with Dr. György Miklós, Dr. András Rácz and Dr. Andras Valkó on Mobile IP protocol testbed implementation and the design of ad hoc network protocols for the Bluetooth short-range wireless technology. From 2001 to 2007, he worked in the group of Prof. JeanPierre Hubaux in the Laboratory of Computer Communications and Applications at EPFL. During his stay at EPFL, he worked on incentives for wireless social community networks, on reputation systems based on game theory to avoid denial of service in wireless ad hoc networks and on incentive-aware network design for various wireless networks. Since 2006, he is an associate member of the Laboratory of Cryptography and Systems Security (CrySyS) at Budapest University of Technology and Economics (BME), Hungary. He collaborates on projects in wireless security and incentive design for wireless networks. In 2008, he was a postdoctoral researcher at the Department of Electrical Engineering and Computer Science at UC Berkeley in the group of Prof. Jean Walrand. At UC Berkeley, he participated in research focusing on incentive design to improve the security of computer systems, in particular to increase user awareness, to manage risks and liabilities. In 2009, Mark joined the security group at the International Computer Science Institute (ICSI) in Berkeley. His current research focuses on measurement-based malware analysis and incentive design to improve Internet security.

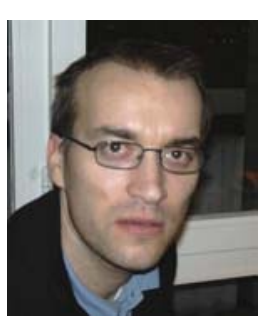

Mario Čagalj received the Dipl.Ing degree in computer science and electrical engineering from the University of Split, Croatia, in 1998, and the $\mathrm{PhD}$ degree in communication systems from the Ecole Polytechnique Federale de Lausanne (EPFL) in February 2006. In 2000 and 2001, he completed the Predoctoral School in Communication Systems, EPFL. From 2001 to 2006, he was a research assistant in the Laboratory for Computer Communications and Applications (LCA) at EPFL. Since September 2006, Mario Cagalj is an Assistant Professor at Faculty of Electrical Engineering, Mechanical Engineering, and Naval Architecture (FESB), University of Split, Croatia. His research interests include the design and analysis of security protocols for wireless networks, applied cryptography, applications of game theory to wireless (and wired) networks, and the design of energy-efficient communication protocols for wireless networks. More details can be found at http://www.fesb.hr/ mcagalj 


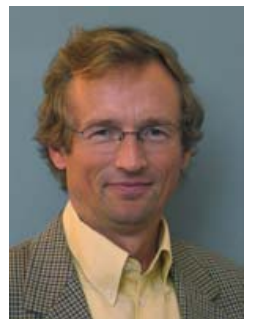

Jean-Pierre Hubaux joined the faculty of EPFL in 1990. His research activity is focused on wireless networks, with a special interest in security and cooperation issues. In 1991, he designed the first curriculum in communication systems at EPFL. He was promoted to full professor in 1996. In 1999, he defined some of the main ideas of the National Competence Center in Research named "Mobile Information and Communication Systems" (NCCR/MICS); this center (still very active nowadays) is often nicknamed "the Terminodes project". In this framework, he has notably defined, in close collaboration with his students, novel schemes for the security and cooperation in wireless networks; in particular, he has devised new techniques for key management, secure positioning, and incentives for cooperation in such networks. In 2003, he identified the security of vehicular networks as one of the main research challenges for real-world mobile ad hoc networks. In 2007, he completed a graduate textbook entitled Security and Cooperation in Wireless Networks, with Levente Buttyan.

$\mathrm{He}$ is co-founder and chairman of the steering committee of WiSec (the ACM Conference for Wireless Network Security). He is also the chairman of the steering committee of MobiHoc (the ACM Symposium on Mobile Ad Hoc Networking and Computing) and a member of the steering committee of IEEE Transactions on Mobile Computing. He has been serving on the program committees of numerous conferences and workshops, including SIGCOMM, INFOCOM, MobiCom, MobiHoc, SenSys, WiSe, and VANET. $\mathrm{He}$ is a member of the Federal Communications Commission (ComCom), the "Swiss FCC."

He held visiting positions at the IBM T.J. Watson Research Center and at UC Berkeley. He is an IEEE Fellow. He was born in Belgium, but spent most of his childhood and youth in Northern Italy. After completing his studies in electrical engineering at Politecnico di Milano, he worked 10 years in France with Alcatel, primarily in the area of switching systems architecture and software. 